\title{
Inventaire Des Nématodes Parasites et Mycorhization Naturelle de l'Igname (Dioscorea spp.) Dans La Région Centrale Du Togo
}

\author{
Kassankogno Abalo Itolou, \\ Institut de l'Environnement et Recherches Agricoles, \\ Bobo-Dioulasso, Burkina Faso
}

Tchabi Atti,

Institut Supérieur des Métiers de l'Agriculture, Université de Kara, Togo

Sokamé Bonoukpoè Mawuko,

International Centre of Insect Physiology and Ecology, Nairobi, Kenya

Sanbonga Raphael Kolani,

Institut de Conseil et d'Appui Technique (ICAT), Cacaveli Lomé, Togo

Haougui Adamou,

Institut National de Recherches Agricoles, Niamey, Niger

Gumedzoe Dieudonné Mawuema Yawovi,

Université de Lomé, Ecole Supérieure d'Agronomie Lomé, Togo

URL:http://dx.doi.org/10.19044/esj.2020.v16n3p436

\section{Résumé}

L'igname, Dioscorea spp. est une culture très importante au Togo avec plusieurs espèces cultivées. Toutefois, le complexe D. cayennensis- rotundata est le plus répandu dans les exploitations. Malheureusement, les nématodes sont des parasites majeurs qui entravent sa production. A cet effet, cette étude a été conduite dans trois préfectures de la région centrale du Togo dans le but d'identifier les nématodes parasites qui font intrusion dans les champs d'igname et d'évaluer la mycorhization naturelle de la plante. Des prélèvements de sol et de racines ainsi que l'inspection des tubercules d'igname pour les dégâts de fissuration et de galles dues aux nématodes ont été effectués à différents endroits de différents champs d'igname dans chaque préfecture. Des échantillons de sol et de racines ramenés au laboratoire, ont été extraits des nématodes et identifiés sur la base de critères morphologiques en utilisant les clés d'identification. Le taux de mycorhization a été évalué par la coloration des racines. Les résultats ont révélé la présence des genres Scutellonema, Meloïdogyne, Aphelenchoides et Xiphinema dans les champs avec Scutellonema spp. et Meloidogyne spp. comme nématodes majeurs sur les ignames. L'évaluation des dégâts visuels des nématodes sur les tubercules 
a montré que les taux de fissures causées par Scutellonema spp. vont de 80 à $100 \%$ alors que les taux de galles causées par Meloidogyne spp. sont de 60 à $70 \%$. Le taux de mycorhization naturelle des plantes d'ignames va de 10 à $86,66 \%$ en milieu naturel de culture en fonction des sites et des cultivars. Cette étude est la première réalisée en milieu paysan au Togo pour inventorier la diversité des nématodes de l'igname. Les résultats de cette étude servent de base à l'élaboration des stratégies de gestion des nématodes pour une production durable de l'igname.

Mots clés : Dioscorea spp, Nématodes, parasites, Mycorhizes, Togo

\title{
Inventory of Parasitic Nematodes and Cultivated Plants Natural Mycorrhization of Yam (Dioscorea Spp.) in Central Region of Togo
}

\author{
Kassankogno Abalo Itolou, \\ Institut de l'Environnement et Recherches Agricoles, \\ Bobo-Dioulasso, Burkina Faso
}

Tchabi Atti,

Institut Supérieur des Métiers de l'Agriculture, Université de Kara, Togo

Sokamé Bonoukpoè Mawuko,

International Centre of Insect Physiology and Ecology, Nairobi, Kenya

Sanbonga Raphael Kolani,

Institut de Conseil et d'Appui Technique (ICAT), Cacaveli Lomé, Togo

Haougui Adamou,

Institut National de Recherches Agricoles, Niamey, Niger

Gumedzoe Dieudonné Mawuema Yawovi, Université de Lomé, Ecole Supérieure d'Agronomie Lomé, Togo

\begin{abstract}
Yam, Dioscorea spp. is an important crop in Togo with several cultivated species. However, D. cayennensis- rotundata complex is the most widespread in the exploitations. Unfortunately, the nematodes are major pests that hindered its production. Therefore, this study was conducted in three different counties in central region of Togo. The objectives were to identify the parasitic nematodes associated with yam crop, to evaluate the damage of these parasitic and the natural mycorhization of the yam. Soil and root were
\end{abstract}


sampled and inspection of yam tubers for nematode cracking and gall damage were done at different locations of different yam fields in each county. From soil and root samples taken into the laboratory were extracted the nematodes and identified based on morphological criteria using the identification keys. The rate of mycorrhization was assessed by staining of the roots. The results revealed the presence of the genus Scutellonema, Meloidogyne, Aphelenchoides and Xiphinema in the fields and showed that Scutellonema spp. and Meloidogyne spp. are the major parasitic nematodes of yam in the areas of the study. The assessment of the visual damages of the nematodes on the tubers showed that the crack rates due to Scutellonema spp. were between 80 and $100 \%$ while the gall rates due to Meloidogyne spp. varied between 60 and $70 \%$. The frequency of natural mycorhization of yams ranging from 10 to $86,66 \%$ and is related to the site and the variety. This study could be the first carried out in Togo to inventory the nematodes diversity of the yam. These results will contribute to nematodes management strategies elaboration for durable production of yam.

Keywords: Dioscorea spp, Nematodes, parasitics, Mycorhizes, Togo

\section{Introduction}

L'igname, Dioscorea spp. est le plus important tubercule cultivé pour l'alimentation en Afrique de l'Ouest. Elle représente un important aliment de base des petits exploitants agricoles, particulièrement aux Benin, Nigeria et Togo (Ijoyah, 2011; Ile et al., 2007). Plus de $90 \%$ de la production globale (50 millions de tonnes de tubercules fraiches/an) est assurée par l'Afrique de l'Ouest (Nweke, 2015; Ravi et al., 1996). Dans cette région, des millions de personnes tirent leur supplément alimentaire de l'igname et elle leur fournit un tiers des calories essentielles nécessaires (Nweke et al., 1992). Elle est donc considérée comme une culture vitale pour améliorer la sécurité alimentaire dans la région (Bricas \& Attaie, 1997; Nindjin et al., 2007). Cette culture continuera de jouer un rôle majeur dans les systèmes alimentaires des pays en développement parce qu'elle s'adapte à une utilisation diverse. Dans de nombreux pays, en particulier au Bénin et au Togo, il existe un écart croissant entre les niveaux de l'offre et de demande de l'igname (Afouda et al., 2004; Houedjissin \& Koudande, 2010). Alors que la demande pour l'igname croît, la production et la productivité sont à la baisse. Le rendement atteint varie de 8 à 12 t/ha (Ettien et al., 2003; N'Goran et al., 2007), tandis que le potentiel est d'environ 65 t / ha (FAO, 1999). Ceci est dû au système de production qui priorise la subsistance, aux coûts de production élevés et à la nécessité de l'amélioration et le maintien de la qualité des terres agricoles. Les carences et les déséquilibres nutritionnels sont principalement des obstacles à une production agricole durable en Afrique de l'Ouest (Ojeniyi, 1990). Bien 
qu'ayant un potentiel de rendement élevé, de faibles rendements de l'igname sont régulièrement observés et ceci a été souvent attribué à un faible niveau de fertilité des sols et des attaques de ravageurs principalement les nématodes parasites. Il a été rapporté des pertes de tubercules d'igname de plus de $25 \%$ entre la récolte et la vente due aux nématodes, aux insectes ravageurs et aux maladies (Egesi et al., 2006; Ezeh, 1998). Parmi les nématodes associés à l'igname, trois groupes d'espèces suivants sont considérés comme contraintes majeures : nématode de l'igname (Scutellonema bradys), nématode de racine (Meloidogyne spp.) et nématodes de lésion (Pratylenchus coffeae and $P$. sudanensis) (Bridge et al., 2005). Dans les tubercules d'igname commercialisés sur les marchés de l'igname en Afrique de l'Ouest, S. bradys et Meloidogyne spp. sont les principaux nématodes avec $2.84 \%$ de tubercule d'igname infectés par S. bradys et $2.94 \%$ infectés par Meloidogyne spp. (Coyne et al., 2006). Des résultats similaires ont été reportés au Ghana (Jamani et al., 2016).

Les pesticides peuvent être utilisés pour lutter contre les nématodes, mais ils sont coûteux, indisponibles ou hautement toxiques pour l'homme et l'environnement, ce qui constitue un risque sérieux pour la santé humaine et environnementale (FAOSTAT, 2007). D'autres pratiques de gestion des nématodes, telles que le traitement des tubercules à l'eau chaude (IIITA 2006), l'utilisation de plantes de couverture (Claudius-Cole, 2005), des plantes pièges(El-Nagdi \& Youssef, 2004), des engrais chimiques (Baimey, 2005) ou organiques (Morais et al., 2016) ont été explorées pour l'igname mais qui ne sont toutefois pas effectives pour réduire les dégâts au-dessous du seuil économique. Les progrès récents de la biotechnologie ont également montré que la culture tissulaire de l'igname fournirait du matériel végétal exempt de parasites et de maladies, en utilisant des techniques de culture aseptique in vitro de méristème et de bout de pousse (IIITA, 2006). Cependant, les vitroplants obtenus sont délicats et fragiles, et non seulement exempts d'agents pathogènes, mais également exempts de tous les micro-organismes bénéfiques naturels, tels que les champignons mycorhiziens arbusculaires (CMA).

En Afrique de l'Ouest, des études récentes ont montré une importante biodiversité des espèces des CMA et leur potentiel de promotion de la croissance de l'igname (Tchabi et al., 2009, 2008, 2010), la suppression des nématodes et l'amélioration du rendement des systèmes de production (Affokpon et al., 2011).

Les CMA sont des éléments importants de la microflore du sol dans les agroécosystèmes, qui forment une symbiose mutualiste avec la plupart des espèces végétales, y compris la quasi-totalité des plantes actuellement micro propagées (Smith \& Read, 2008). Les CMA sont actifs dans l'augmentation de la disponibilité et de l'absorption du phosphore et des oligo-éléments du sol, améliorant ainsi la croissance des plantes hôtes (Dodd, 2000; Hamel, 
1996). Ils peuvent atténuer les stress biotiques et abiotiques (Alarcon et al., 2008; Audet \& Charest, 2007; Davies et al., 2001). Ils sont également particulièrement importants dans les systèmes d'agriculture durable où les intrants non biologiques sont faibles (Brussaard et al., 2007). En général, la colonisation des racines favorise le développement des plantes en augmentant l'absorption des nutriments, l'activité hormonale, le taux de croissance et donc le rendement (Arriagada et al., 2007; Smith \& Read, 2008), mais aussi la suppression des pathogènes et de nématodes parasites (Hol \& Cook, 2005; Ryan \& Angus, 2003; Vestberg \& Kukkonen, 2004). Au Togo, aucune étude n'a encore été rapportée sur le statut des espèces de nématodes associés à l'igname et l'étendue de l'association naturelle de l'igname avec les CMA en termes de gestion des nématodes et une production durable de l'igname à ce jour (Ahulu et al., 2005; Dare et al., 2007).

Cette présente étude investigue sur la diversité des espèces de nématodes associés à l'igname dans trois différentes préfectures de la région centrale du Togo ainsi que son niveau de mycorhization naturelle.

\section{Materiel Et Methodes}

\subsection{Site d'expérimentation}

La présente étude a été réalisée dans la région centrale du Togo (Fig. 1). Cette région est la zone où l'igname est beaucoup plus cultivée dans le pays. C'est une zone au climat de type soudano-guinéen avec un régime de pluie monomodal. La pluviométrie varie de 1000 à $1300 \mathrm{~mm}$. Les échantillons ont été prélevés sur onze (11) sites différents dans les champs des paysans, répartis dans trois (3) préfectures à savoir Sotouboua, Tchamba et Tchaoudjo en deux périodes différentes, août et novembre 2018. Les sites de prélèvement ont été choisi de façon aléatoire. 


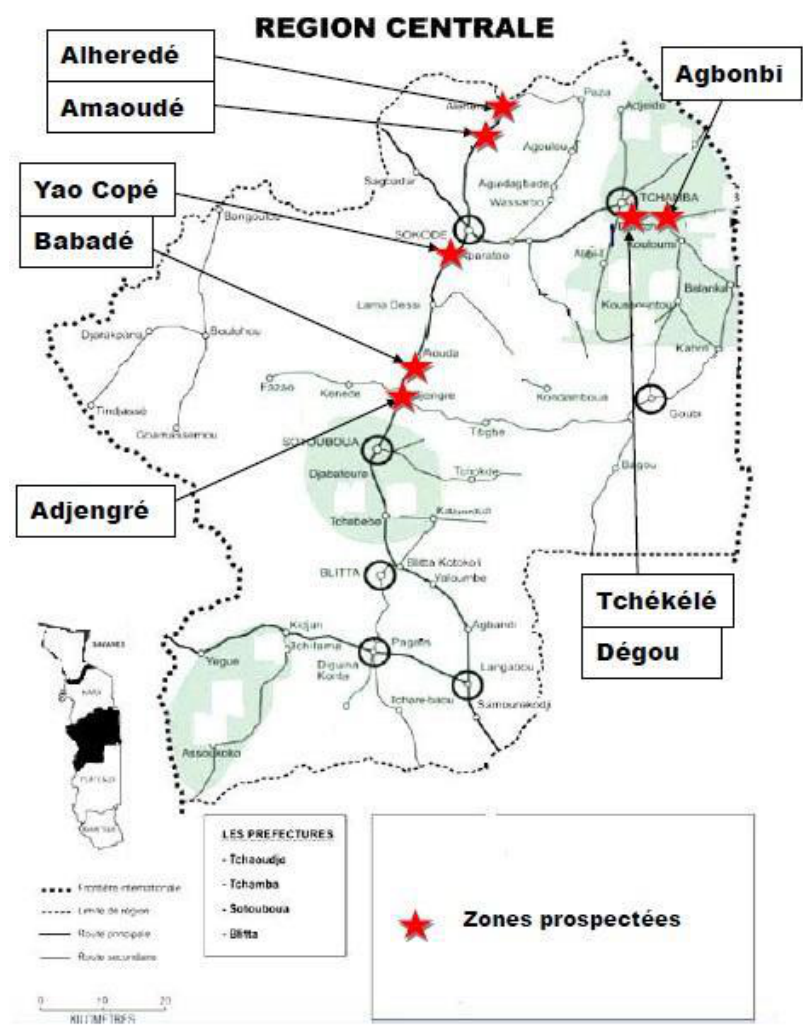

Figure 1: Carte de la localisation de la zone d'étude

\subsection{Technique d'échantillonnage}

Dans les champs prospectés, les échantillonnages ont été fait suivant les diagonales. Le sol a été prélevé à l'aide d'une sonde à une profondeur de 15 à $20 \mathrm{~cm}$ dans la rhizosphère des plantes d'igname. Le système racinaire contenu dans l'échantillon ainsi prélevé constitue l'échantillon de racine. Dans chaque champ, quatre (4) prélèvements ont été effectués (200 g chacun) à différents endroits de façon aléatoire. Ces quatre prélèvements ont été malaxés selon la méthode décrite par Barker \& Niblack (1990) en utilisant un sachet plastique pour constituer un échantillon composite pour une unité expérimentale. Au total 50 échantillons ont été collectés.

\subsection{Technique d'extraction des nématodes}

Pour l'extraction, la méthode de Baermann modifiée (Coyne et al., 2007) a été utilisée. Cette adaptation de la méthode de Baermann vise à pallier le manque d'aération de la méthode d'origine et son principe est le suivant : 5 $\mathrm{g}$ de racine ou $100 \mathrm{~g}$ de sol, sont prélevés selon que l'on veut extraire les nématodes des racines ou du sol, puis mis dans un tamis tapissé à l'intérieur par du papier hygiénique (jouant le rôle de filtre) et le tout est placé dans une bassine en plastique. Chaque échantillon est bien éparpillé dans le tamis à 
l'aide d'une pincette. Ensuite l'eau a été ajoutée jusqu'à couvrir légèrement l'échantillon de sols ou de racines, favorisant ainsi la migration des nématodes des échantillons de sols et de racines vers l'eau qui constitue le milieu d'extraction. Il faut noter que pour les racines celles-ci sont bien nettoyées à l'eau de robinet, découpées et broyées à l'aide d'un Moulinex avant leur transfert dans le tamis. Les échantillons ainsi préparés sont laissés incubés pendant $24 \mathrm{~h}$ pour le sol et $48 \mathrm{~h}$ pour les racines à $25^{\circ} \mathrm{C}$ dans une chambre noire. Après $24 \mathrm{~h}$ ou $48 \mathrm{~h}$, on récupère le milieu d'extraction dans une éprouvette graduée. Après $30 \mathrm{mn}$ de décantation du milieu, on fait diminuer le surnageant avec précaution à l'aide d'une trompe à vide pour ramener le niveau du milieu à $100 \mathrm{ml}$ dans l'éprouvette pour des raisons de commodité avant de passer à l'observation à la loupe.

\subsection{Comptage des nématodes}

Les nématodes du sol ou de racines ont été dénombrés sous une loupe binoculaire. Le dénombrement a été fait à l'aide d'une boîte de Pétri à fond quadrillé de $15 \mathrm{ml}$ de contenance. Les $100 \mathrm{ml}$ du milieu extraction préalablement constitués dans l'éprouvette graduée ont été bien agité et $10 \mathrm{ml}$ de suspension ont été prélevés à l'aide d'une pipette graduée dans la boîte de Pétri à fond quadrillé puis placés sous la loupe binoculaire pour l'observation et comptage des nématodes. Trois observations de chaque échantillon ont été faites. Les valeurs de nématodes comptées de chaque observation ont été ramenées à $100 \mathrm{~g}$ de sol ou $100 \mathrm{~g}$ de racines pour les analyses. Les nématodes observés ont été fixés à partir d'un mélange à égal volume de l'éthanol $95^{\circ}$ et de la glycérine pure, le tout porté à une température de $70^{\circ} \mathrm{C}$ pour l'identification.

\subsection{Identification des nématodes}

Avant de procéder à l'identification, les nématodes préalablement fixés sont d'abord placés dans des blocs (stain blocks) et puis dans un microfour à $40^{\circ} \mathrm{C}$ pendant $72 \mathrm{~h}$. Ce procédé permet de faire évaporer l'alcool et de laisser seulement la glycérine. Ensuite les nématodes sont prélevés et montés entre lame et lamelle. L'identification s'est faite au microscope (Motic, Model B1) à X4, X10, X40 et X100 sur la base de critères morphologiques en utilisant les clés d'identification (Siddiqi, 2000).

\subsection{Distribution des genres de nématodes}

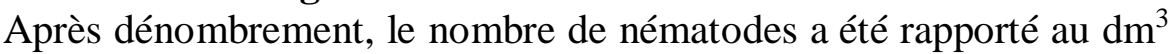
de sol humide pour les échantillons du sol et au gramme de racine sèche pour les échantillons de racine. La fréquence et l'abondance de chaque genre des nématodes sont déterminées à partir du diagramme de Fréquence/Abondance (Fortuner \& Merny, 1973): 
La fréquence $(\boldsymbol{F})$ est le pourcentage d'échantillons qui contiennent le nématode considéré :

$\mathrm{F}=(\mathrm{e} / \mathrm{n}) \times 100$, avec e : nombre d'échantillons contenant le genre considéré et $\mathrm{n}:$ nombre total d'échantillons.

L'abondance (A) d'un genre de nématode est la moyenne des densités des échantillons dans lesquels le genre a été retrouvé.

Elle est calculée sous la formule suivante : $\mathbf{A}=\boldsymbol{\Sigma} \mathbf{X i} / \mathbf{e}$, avec $\mathbf{X i}=$ nombre $\mathrm{d}$ 'individus du genre considéré par $\mathrm{dm}^{3}$ de sol ou par gramme de racines sèches et $\mathbf{e}=$ nombre d'échantillons dans lesquels le genre considéré est présent.

Un genre est donc abondant et fréquent lorsqu'il est présent dans au moins $30 \%$ des échantillons avec au moins 200 individus par dm3 de sol ou 20 individus par gramme de racine sèche. En dessous de ces valeurs, il est considéré peu fréquent et peu abondant. Cependant, un genre de nématode peut être considéré fréquent et peu abondant ou vis versa.

\subsection{Evaluation des dégâts de quelques genres de nématodes sur les tubercules}

L'évaluation a porté sur les dégâts causés par Scutellonema spp. caractérisés par les symptômes de pourriture sèche et fissurations et ceux causés par Melö̈dogyne spp. caractérisés par la présence des galles. Pour ce faire, l'échelle de cotation de 1-5 de Claudius-Cole (2005) a été utilisée. La fréquence de chaque type de dégât a été calculée en fonction des sites.

\subsection{Evaluation des fréquences de mycorhization des plantes au champ}

Le taux de mycorhization a été évalué selon la méthode de Phillips \& Hayman (1970) par la coloration des racines. Pour chaque plant, 3 lames de 10 fragments ont été préparées puis examinées sous microscope au grossissement x100. La fréquence (F) des racines colonisées a été évaluée en utilisant la formule ci-dessous (Djigal, 2003).

Fréquence $(F)=($ Nombre de fragments colonisés/ Nombre de fragments observés) x 100

\subsection{Analyses statistiques}

L'analyse statistique a porté sur la densité de nématodes dans le sol et de racines en fonction des sites prospectés et des cultivars d'ignames ainsi que les dégâts de l'attaque par Scutellonema spp. et Melö̈dogyne spp. Toutes les données collectées ont été analysées avec le logiciel Genstat discovery $3^{\text {rd }}$ éd. par une analyse de la variance (ANOVA) et les moyennes sont discriminées à l'aide des tests de Student Newman Keuls (SNK) au seuil de 5\%. Les densités moyennes des nématodes ont été normalisées en utilisant la transformation $\log 10(\mathrm{x}+1)$ (où $\mathrm{X}$ est la densité moyenne de nématodes), avant l'analyse. 


\section{$3 \quad$ Résultats}

\subsection{Densité de nématodes dans le sol et de racines des différents cultivars d'igname}

Les résultats de l'analyse ont montré une différence hautement significative entre les densités moyennes de nématodes dans les différents sols analysés au cours des prospections aussi bien dans la période d'Aout $(F=$ 124.5; $d f=9 ; P<0,001)$ que celle de Novembre $(F=77.25 ; d f=10 ; P<$ 0,001) (Fig. 2 A et B). La discrimination des moyennes en fonction des sites a montré qu'au cours de la première prospection les sites de Kpario, Alheredé A et Alheredé B sont beaucoup plus infestés par rapport aux autres sites. La deuxième période de prospection a donné les mêmes résultats que celle de la précédente mais avec une légère diminution de la densité moyenne de nématodes sur les sites.
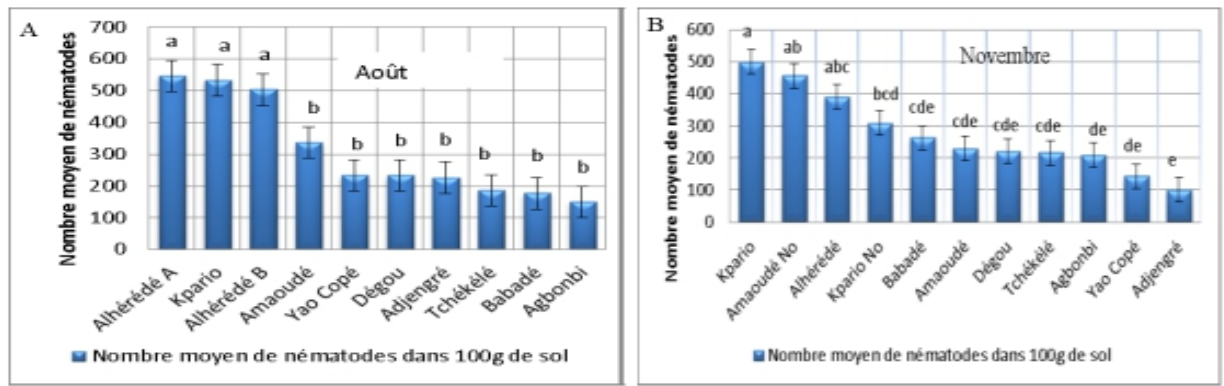

Figure 2: Nombre moyen de nématodes dans $100 \mathrm{~g}$ de sol en période d'Août (A) et Novembre (B). Les données ont été reportées comme moyenne and erreur standard pour trois répétitions. Les différences non significatives entre les traitements sont montrées par des lettres identiques déterminées à l'aide du test de Student Newman Keuls (SNK) au seuil de $5 \%$ précédé de l'analyse de la variance (ANOVA).

$\mathrm{Au}$ cours de la prospections, quatre (4) cultivars d'igname ont été répertoriés à savoir Katala encore appelé Hè-abala, Brutani, Labaco et Alassora. Dans les racines, l'analyse de variance a montré qu'il existe une différence statistiquement significative $(F=27,33 ; d f=3 ; P=0,002)$ entre les attaques des cultivars Katala, Hè-abala, Brutani et labaco contre le cultivar Alassora qui est moins sensible à l'attaque des nématodes. Les données de l'extraction des nématodes dans les racines des différents cultivars en fonction des sites ont montré une forte infestation des cultivars sur le site de Kpario (en moyenne 4000 nématodes pour $100 \mathrm{~g}$ de racine) par rapport aux autres sites qui ont montré des infestations comprises entre 500 et 2000 nématodes pour $100 \mathrm{~g}$ de racines (Fig. 3). Cependant, le cultivar Alassora n'a montré aucune différence significative $(F=2,04 ; d f=6 ; P=0,82)$ entre les attaques des plantes selon les sites où la présence de nématodes a été révélé (Fig. 3 ). 

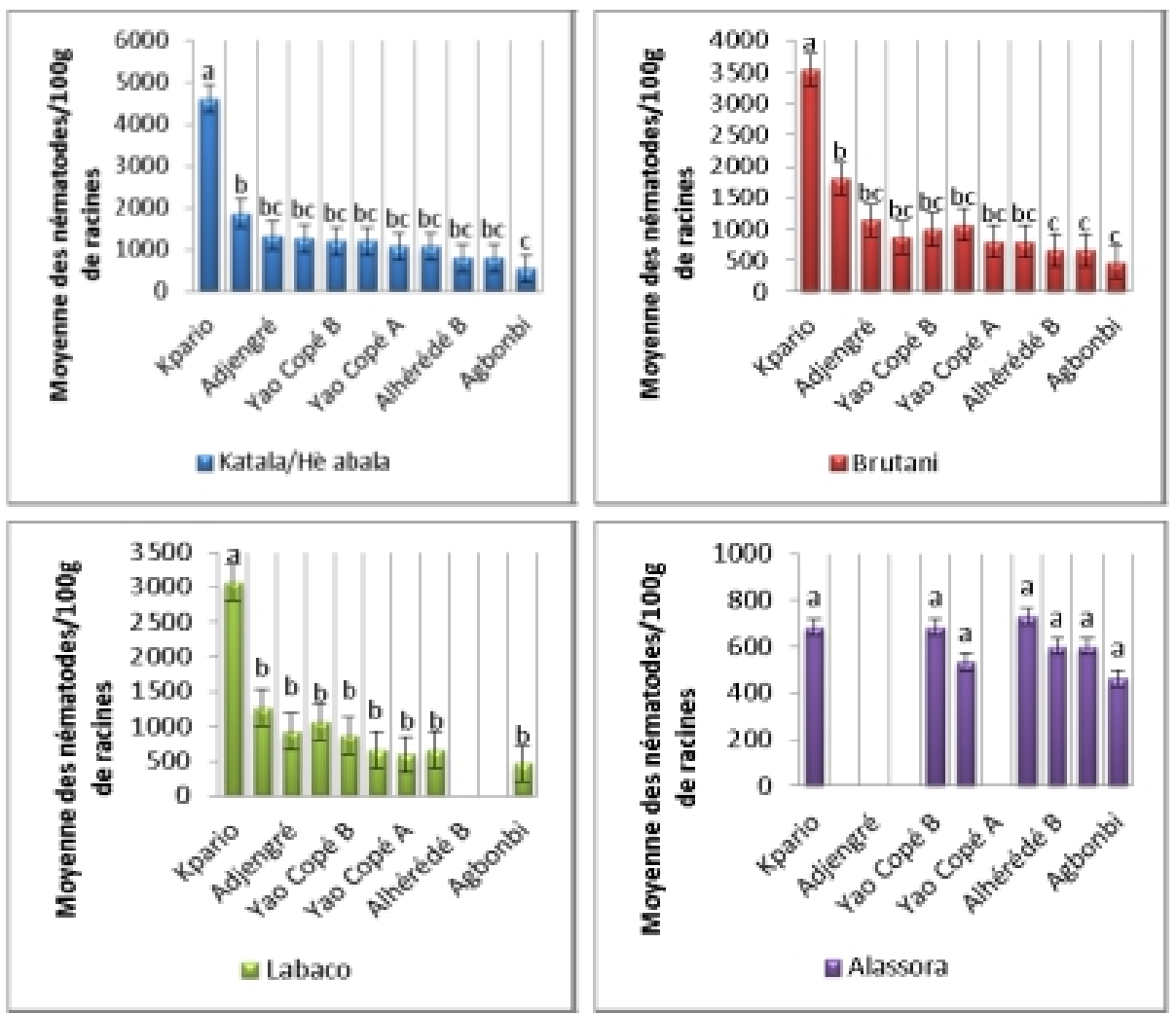

Figure 3: Densité moyenne des nématodes dans les racines de 4 cultivars. Les données ont été reportées comme moyenne and erreur standard pour trois répétitions. Les différences non significatives entre les traitements sont montrées par des lettres identiques déterminées à l'aide du test de Student Newman Keuls (SNK) au seuil de 5\% précédé de l'analyse de la variance (ANOVA).

\subsection{Diversité, fréquence et abondance des communautés de nématodes parasites associes à l'igname}

Les résultats de l'identification des nématodes du sol et des racines issues des sites prospectés dans les préfectures de Tchaoudjo, Tchamba et Sotouboua sont présentés dans le Tableau1. Les nématodes aussi bien phytoparasites que saprophages ont été identifiés.

Quatre (4) genres de nématodes parasites d'ignames ont été identifiés sur l'ensemble des sites à savoir les genres Meloidogyne, Scutellonema, Xiphinema et Aphelenchoides. Ils sont repartis en quatre (4) familles ; deux (2) ordres et une (1) classe (Tableau. 1). D'une façon générale l'analyse a révélé la présence d'un endoparasite sédentaire (Meloidogyne spp), d'un endoparasite migrateur (Scutellonema spp), de deux ectoparasites (Xiphinema spp et Aphelenchoides spp). Par ailleurs, certaines espèces de nématodes appartenant aux ordres des Rhabditida et des Enoplida mais le plus souvent saprophytes ont été également identifiés. 
Tableau 1: les nématodes identifiés sur les différents sites prospectés

\begin{tabular}{|c|c|c|c|c|}
\hline Classe & Ordre & Sous-ordre & Famille & Genre \\
\hline \multirow{4}{*}{ Secermentea } & \multirow{3}{*}{ Tylenchida } & Tylenchina & Hoplolaimidae & Scutellonema \\
\hline & & & Heteroderidae & Meloidogyne \\
\hline & & Aphelenchina & Aphelenchoidae & Aphelenchoides \\
\hline & Dorylaimida & Dorylaimina & Longidoridae & Xiphinema \\
\hline
\end{tabular}

L'abondance et la fréquence de chacun des genres de nématodes phytoparasites identifies varient selon les zones préfectorales et selon que cela soit dans le sol ou des racines. Dans la préfecture de Sotouboua, un ectoparasite (Xiphinema) identifié dans le sol et un endoparasite (Meloidogyne) retrouvé aussi bien dans le sol que dans les racines apparaissent tous deux fréquents et abondants (Figure 4 A et B). Dans la préfecture de Tchamba, les deux endoparasites identifiés apparaissent fréquents et abondants dans les racines. Par contre seul Scutellonema est fréquent et abondant dans le sol et Meloidogyne peu fréquent (moins de 30\%) et peu abondant (moins de 200 individus par $\mathrm{dm}^{3}$ ) (Figure $4 \mathrm{C}$ et D). Dans la préfecture de Tchaoudjo, Meloidogyne, Scutellonema sont fréquents (plus de $30 \%$ ) et abondant (plus de 200 individus par $\mathrm{dm}^{3}$ ) aussi bien dans le sol que dans les racines (Figure $4 \mathrm{E}$ et $\mathrm{F}$ ). Par ailleurs, Xiphinema retrouvé uniquement dans le sol et Aphelenchoides uniquement dans les racines sont peu fréquents (moins de 30\%) et peu abondants (moins de 200 individus par $\mathrm{dm}^{3}$ ). 


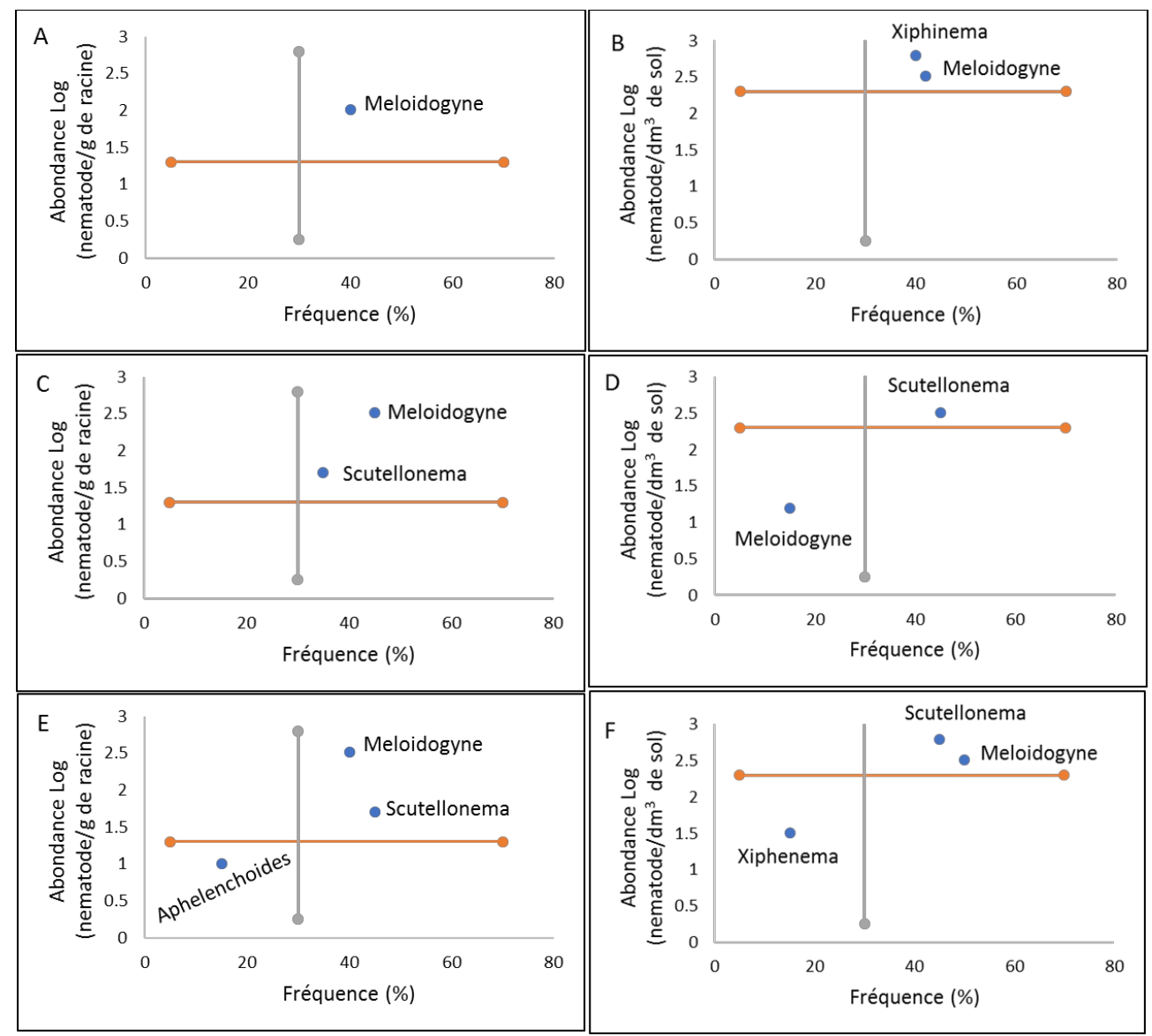

Figure 4: Fréquence et abondance des genres de nématodes parasites de l'igname observés dans les racines et dans le sol dans les préfectures de Sotouboua (A\&B), Tchanba (C\&D) et Tchaoudjo (E\&F)

\subsection{Evaluation des symptômes visuels de Scutellonema spp et de Meloüdogyne spp \\ L'évaluation des symptômes de fissures (crackings) et de galles sur les} tubercules dans les champs prospectés a montré que le taux d'infection par Scutellonema spp. (fissures) est plus important (80 à 100\%) que les galles de Meloidogyne spp. (<70\%) (Fig. 5). Au total 240 tubercules évalués et plus de $92 \%$ présentent des symptômes de fissuration contre $27,5 \%$ pour les symptômes de galles. 

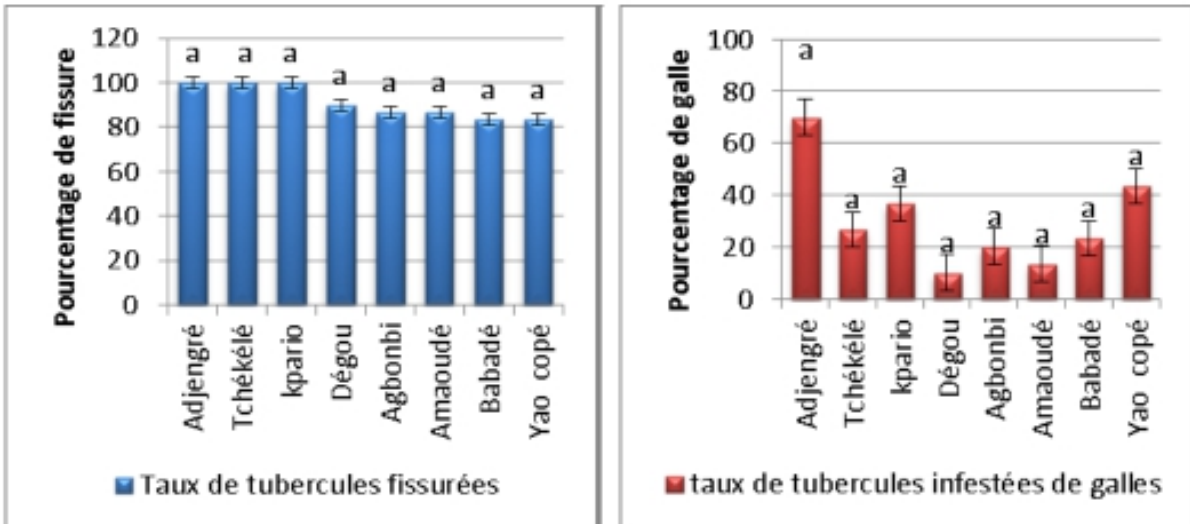

Figure 5: Le taux de fissures et de galles sur tubercules par sites. Les données ont été reportées comme moyenne and erreur standard pour trois répétitions. Les différences non significatives entre les traitements sont montrées par des lettres identiques déterminées à l'aide du test de Student Newman Keuls (SNK) au seuil de 5\% précédé de l'analyse de la variance (ANOVA).

\subsection{Taux de mycorhization naturelle des cultivars d'ignames sur les sites prospectés}

Les résultats de l'analyse de la fréquence de mycorhization naturelle des plantes d'igame (cultivars) sur les différents sites sont présentés dans la Fig. 6.

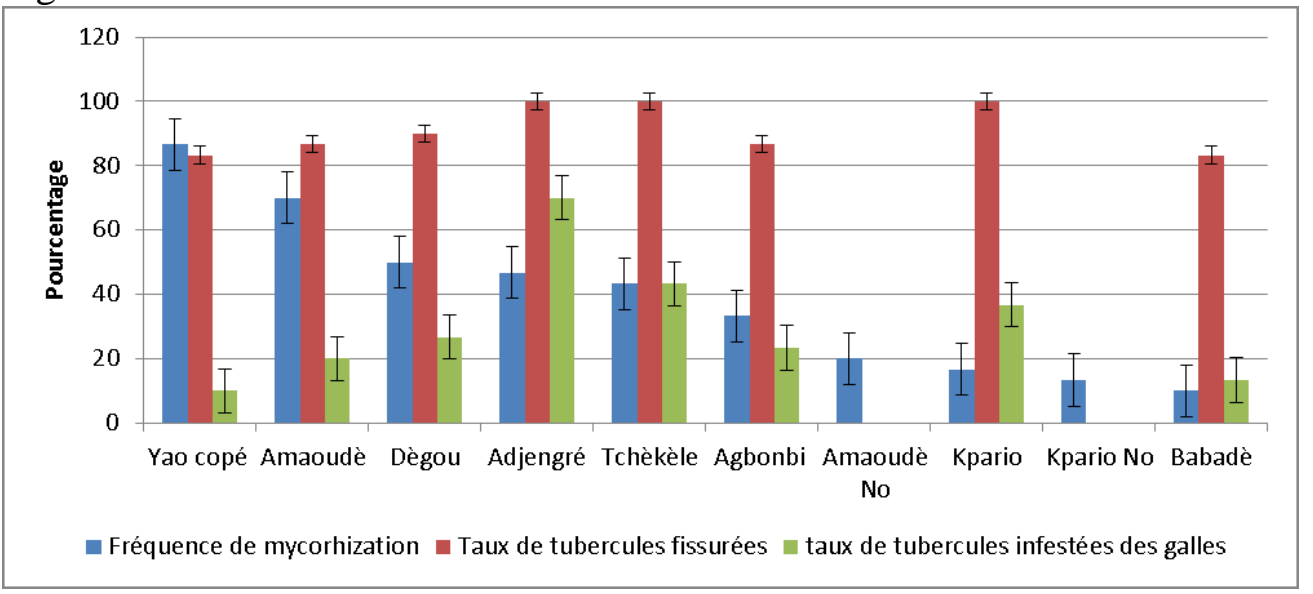

Figure 6: Comparaison du taux de mycorhization en fonction des symptômes de fissures et de galle sur chaque site.

Ces résultats ont révélé une fréquence très élevée de mycorhization des plantes sur les sites de Yao Copé et d'Amaoudé $(P<0.001)$. En revanche, les sites de Dégou, Adjengré, Tchékélé, Alheredè et Agbonbi sont moyennement mycorhizés. Sur ces derniers sites la fréquence de mycorhization est comprise entre 30 et $50 \%$. 
Les fréquences de mycorhization enregistrée varient en fonction des sites et en fonction des cultivars. En effet les fréquences de mycorhization élevées sont enregistrées sur le cultivar Katala/Hè-abala où elles sont respectivement de $86,66 \%$ et $70 \%$. Les cultivars Monya et Labaco ont montré un taux faible de mycorhization (moins de $30 \%$ de racines mycorhizées).

L'analyse corrélative des fréquences de mycorhization et des attaques racinaires par les nématodes représentés par le nombre moyen de nématodes dans les racines des plantes par site montre que les sites à faible colonisation mycorhizienne des plantes présentent une forte attaque racinaire due aux nématodes. Les sites de Babadè et Kpario en témoignent de cette constatation avec des fréquences de mycorhization respectivement de $10 \%$ et $16,66 \%$ et des attaques racinaires dépassant les 1500 nématodes pour $100 \mathrm{~g}$ de racines. Les analyses corrélatives entre les taux de mycorhization et les symptômes de fissures ou de galles sur les tubercules sont présentées dans la Fig. 6. Ces analyses ont montré que les sites fortement mycorhizés présentent de faibles dégâts des nématodes et ceci surtout pour les symptômes dus à Meloidogyne spp.

\section{Discussions}

Cette étude sur l'inventaire des nématodes parasites des ignames dans les champs des paysans vient compléter celle menée sur les tubercules vendus sur les marchés (Coyne et al., 2006) et pourrait être la première réalisée au Togo auprès des paysans en milieu champ.. Les résultats concernant la diversité des nématodes ont permis d'identifier quatre (4) genres de nématodes parasites des ignames sur les 11 sites ayant fait l'objet de l'étude. Au regard de ces résultats, il ressort que la diversité biologique a été relativement faible sur les différents sites étudiés, ceci par comparaison aux travaux de Adegbite et al. (2006) qui ont travaillé sur les nématodes associés à la culture d'ignames dans les états d'Edo, Ekiti et Oyo au Nigeria et qui ont trouvé un nombre beaucoup plus élevé de genres de nématodes parasites de cette plante. Notons que parmi les 4 genres identifiés figurent les deux principaux genres les plus dommageables en culture d'ignames à savoir Scutellonema et Meloidogyne. Ceux-ci représentent tous deux plus de 50\% de tous les nématodes identifiés. Des nématodes appartenant aux genres Xiphinema et Aplelenchoides sont aussi présents sur les sites, mais moins abondants et peu fréquents. Nos résultats sont comparables à ceux rapportés par Cadet et Mateille (1983) sur cultures vivrières au Togo. De même, ces résultats concordent avec ceux de Quénéhervé, (1998) et de Castagnone-Sereno (1989) qui ont travaillé respectivement en Martinique et en Guyane sur les nématodes parasites des ignames. En effet ces auteurs ont signalé dans leurs travaux que Scutellonema bradys et Meloidogyne spp étaient les principaux nématodes les plus dommageables à la culture d'ignames. Le résultat concernant l'identification 
du genre Xiphinema sur les sites n'a rien de surprenant, car ce genre est souvent identifié comme nématode associé à la culture de la patate douce et du riz (Luc \& Coomans, 1992). Or la culture d'ignames est souvent en rotation avec la patate douce et le riz. La présence des nématodes du genre Aphelenchoides confirme les résultats Degras \& Kermarrec (1976) qui ont signalé la présence de ce genre sur Dioscorea spp. en Guyane. L'identification dans les extraits de racines serait une pollution de l'extraction des racines, car c'est un ectoparasite de bourgeons et feuilles.

Scutellonema spp est plus abondant que Meloidogyne spp mais au niveau des racines ce sont les populations de Meloidogyne qui occupent la grande partie. Ceci paraît juste du fait que les individus du genre Meloidogyne passent une grande partie de leur vie dans les racines des plantes. De même, il est montré que les juvéniles (J2) de Meloidogyne spp. une fois libérés dans le sol, infectent directement les racines où ils complètent leur cycle de vie. Au vu des résultats de fréquences et abondances des divers genres, il se révèle des effets compétitifs entre les populations de ces genres. En effet, lorsque les populations de Scutellonema spp sont importantes dans une structure de la plante, on note une faible population de Melö̈dogyne spp., ce qui pourrait être dû à une inhibition réciproque. En générale, l'infestation est importante sur les sites et ceci est probablement la conséquence de la pauvreté des sols en matière organique et autres éléments indispensables au bon développement et à la vigueur de la plante.

Les résultats concernant le scoring des dégâts des nématodes, principalement ceux de Scutellonema spp. et Meloidogyne spp. ont montré que les symptômes visuels sur les tubercules dus à Scutellonema spp. sont les plus importants sur la plupart des sites. On note un taux de plus de $80 \%$ pour les symptômes de Scutellonema contre moins de $70 \%$ pour ceux de Meloidogyne. L'analyse générale permet de dire que les dégâts de Scutellonema spp. sont les plus importants en culture d'ignames. Ces résultats rejoignent ceux de Coyne et al. (2006) qui ont travaillé sur les tubercules des marchés de divers pays de l'Afrique de l'Ouest sur l'infection de Scutellonema bradys et Meloidogyne spp aux ignames cultivées ainsi que leurs incidences. Cependant, les taux d'infection semblent être plus élevés. D'une façon générale, les dégâts de Scutellonema spp. (nématode endoparasite migrateur) responsable des pourritures sèches et fissures sur les tubercules sont les plus importants sur les tubercules d'igname.

Pour l'évaluation de la mycorhization naturelle des ignames, une association symbiotique entre certains champignons telluriques et les racines des plantes d'ignames ont montré que l'igname était une plante mycorhizée (Djigal 2003; Tchabi et al., 2009). Les résultats de l'analyse comparative entre la fréquence de mycorhization des plantes, le taux d'infestation des racines et les symptômes sur les tubercules ont montré que lorsque la mycorhization est 
forte les racines sont moins attaquées et les dégâts des nématodes sur les tubercules sont faibles. Ce résultat confirme celui de Djigal (2003) qui a travaillé sur l'interaction entre la communauté microbienne du sol (bactéries et champignons mycorhiziens) et les nématodes bactérivores et a montré que la densité des nématodes diminue significativement en présence des champignons endomycorhiziens Glomus aggregatum et Glomus intraradices par rapport au témoin sans champignon. De même, les résultats de cette étude confirment les travaux antérieurs de Tchabi et al. (2009) réalisés au Bénin qui ont signalé la même corrélation négative entre la fréquence de mycorhization et la densité des nématodes. Il faut noter aussi que les taux de mycorhizations étaient fonction des sites et des cultivars mis en culture. En effet les cultivars Hè-abala, Katala et Brutani ont montré une fréquence de mycorhization élevée comprise entre 50 et $87 \%$. Ceci serait probablement la conséquence d'un déficit de minéraux indispensables au développement et à la vigueur de la plante. Ce qui obligeait la plante à développer une importante symbiose mycorhizienne afin d'augmenter le flux important de carbone organique et de minéraux (azote, phosphore, eau, ...) via la profondeur du sol.

L'analyse comparative des taux de mycorhization et le taux moyen de nématodes dans les racines a montré que les racines ayant une forte colonisation mycorhizienne sont moins attaquées par les nématodes. Ces résultats confirment ceux de Tchabi et al. (2008), Djigal (2003), Francl (1993) et Hussey \& Roncadori (1982). Par ailleurs, d'autres études ont trouvé que plusieurs facteurs d'ordre environnementaux sont limitants pour l'efficacité de la symbiose mycorhizienne (Senghor, 1998). Hussey \& Roncadori (1982) ont trouvé que l'efficacité de la mycorhization pouvait aussi être remise en cause par les nématodes lorsque ces derniers colonisent les premières racines de la plante et que l'association mycorhize-plante n'est pas toujours symbiotique.

\section{Conclusion}

$\mathrm{Au}$ terme de cette étude réalisée dans les champs paysans dans trois préfectures de la région centrale du Togo, il ressort que les nématodes parasites des ignames sont plurispécifiques avec quatre (4) genres identifiés. Cependant les genres Scutellonema et Meloidogyne sont les plus importants sur les sites et constituent également les plus dommageables. D'autres genres tels que Xiphinema, Aphelenchoides sont aussi présents dans les champs mais à des populations peu abondantes et moins fréquentes. Ainsi, il convient de dire que pour toutes approches ou stratégies de lutte, l'on devra plus tenir compte essentiellement des genres les plus nuisibles pour la culture tout en ne négligeant pas l'action des autres nématodes. Aussi l'igname est-elle une plante fortement mycorhizée avec une fréquence de mycorhization des racines allant de 10 à 86,66\%. La fréquence de mycorhization est à la fois dépendante 
du cultivar et du milieu. Cet inventaire des nématodes couplé à l'évaluation de la mycorhization naturelle des plants d'ignames doit nous permettre de tenir compte dans l'avenir des potentialités naturelles de la plante et des parasites associés à la plante dans les programmes d'amélioration de cette culture. Ainsi une méthode de lutte efficace doit prendre en compte la diversité des nématodes, leurs incidences et la capacité naturelle (mycorhization) de l'hôte de se défendre contre les parasites. Toutefois, des études ultérieures couvrant les autres régions sont suggérées afin avoir des conclusions globales sur le pays. On pourrait éventuellement, envisager en perspectives une étude sur l'entretien de la symbiose mycorhizienne dans un contexte de lutte biologique.

\section{Remerciements}

Les auteurs tiennent à remercier le Laboratoire d'Entomologie et de Nématologie de l'Ecole Supérieure d'Agronomie, Université de Lomé, spécialement Prof. TOUNOU Agbéko Kodjo pour son assistance.

\section{References:}

1. Adegbite AA, Saka JO, Agbaje GO, Owolade OF, \& Lawal GOOA (2006). Survey of plant-parasitic nematodes associated with yams in Edo , Ekiti and Oyo states of Nigeria . African Journal of Agricultural Research, 1(4), 125-130.

2. Affokpon A, Coyne DL, Htay CC, Agbèdè RD, Lawouin L, \& Coosemans J (2011). Biocontrol potential of native Trichoderma isolates against root-knot nematodes in West African vegetable production systems. Soil Biology and Biochemistry, 43(3), 600-608.

3. Afouda S, Soule, BG, \& Tassou ZF (2004). Etude exploratoire du marche regional des produits a racines et tubercules et de leurs derives. PDRT/LARES, Rapport final.

4. Ahulu EM, Nakata M, \& Nonaka M (2005). Arum- and Paris-type arbuscular mycorrhizas in a mixed pine forest on sand dune soil in Niigata Prefecture, central Honshu , Japan. Mycorrhiza, 15, 129-136. https://doi.org/10.1007/s00572-004-0310-9

5. Alarcon A, Davies FT, Autenrieth RL, \& Zuberer D (2008). Arbuscular Mycorrhiza and Petroleum-Degrading Microorganisms Enhance Phytoremediation of Petroleum-Contaminated Soil. International Journal of Phytoremediation, 10(4), 251-263. https://doi.org/10.1080/15226510802096002

6. Arriagada CA, Herrera MA, Borie F \& Ocampo JA (2007). Contribution of Arbuscular Mycorrhizal and Saprobe Fungi to the Aluminum Resistance of Eucalyptus globulus. Water Air Soil Pollution, 182, 383-394. https://doi.org/10.1007/s11270-007-9349-5 
7. Audet P \& Charest C (2007). Dynamics of arbuscular mycorrhizal symbiosis in heavy metal phytoremediation: Meta-analytical and conceptual perspectives. Environmental Pollution, 147, 609-614. https://doi.org/10.1016/j.envpol.2006.10.006

8. Baimey, HK. (2005). Scutellonema bradys as a pathogen of yam in Benin.(n'exist pas dans le text)

9. Barker KR \& Niblack TL (1990). Soil sampling methods and procedures for field diagnosis. In: Zuckerman BM, editor. Plant nematology laboratory manual. Amherst, MA: The University of Massachusetts Agricultural Experiment Station (pp. 10-19).

10. Bricas N, \& Attaie H (1997). La consommation alimentaire des ignames: Synthèse des connaissances et enjeux pour la recherche. Actes du séminaire international Cirad-Inra-Orstom-Coraf. 3-6 juin 1997, Montpellier, France.

11. Bridge J, Coyne D, \& Kwoseh C (2005). Nematode Parasites of tropical Root and Tuber Crops (Excluding potatoes). In: Luc M, Sikora RA and Bridge $J$ (eds) plant parasitic nematodes in subtropical and tropical agriculture. 2nd Edition, CAB International Publishing. pp. 221-258, 2005 (pp. 793-825).

12. Brussaard L, Ruiter PC De, \& Brown GG (2007). Soil biodiversity for agricultural sustainability. Agriculture, Ecosystems \& Environment, 121, 233-244. https://doi.org/10.1016/j.agee.2006.12.013

13. Cadet P. \& Mateille T., 1983.- Les nématodes parasites des cultures vivrières au Togo. Abidjan, Centre ORSTOM d'Adiopodoumé, 8 p.

14. Castagnone-Sereno, C. (1989). Les nématodes parasites de l'igname (Dioscorea spp.): distribution, action pathogène et moyens de lutte. Accepté à l'Agronomie Tropicale.

15. Claudius-Cole A. (2005). Cover crops in the management of Meloidogyne spp. and Scutellonema bradys on edible yam, studies in Nigeria. Ph.D Thesis Faculty of Agriculture University of Ibadan, Ibadan, Nigeria.

16. Coyne DL, Nicol JM, \& Claudius-Cole B. (2007). Practical plant nematology: A field and laboratory guide.

17. Coyne DL, Tchabi A, Baimey H, Labuschagne N, \& Rotifa I. (2006). Distribution and prevalence of nematodes (Scutellonema bradys and Meloidogyne spp.) on marketed yam (Dioscorea spp.) in West Africa. Field Crops Research, 96, 142-150. https://doi.org/10.1016/j.fcr.2005.06.004

18. Dare M, Fagbola O, \& Asiedu R (2007). Heritability estimates of arbuscular mycorrhizal colonization in Dioscorea species in yam growing regions of Nigeria. In: utilization of diversity in land use 
systems: Sustainable and organic approach to meet human needs. Poster, Tropentag, October 9-11, (p. 2007).

19. Davies FT, Puryear JD, Newton RJ, Egilla JN, \& Grossi JAS (2001). Mycorrhizal fungi enhance accumulation and tolerance of chromium in sunflower (Helianthus annuus). Journal of Plant Physiology, 158, 777-786.

20. Degras L, \& Kermarrec A (1976). Introduction, nématodes et bouturage des ignames", Nouvelles Agronomiques des Antilles et de la Guyane, vol. 2 no. 1 pp. 1-14.

21. Djigal D (2003). Interactions entre la communauté microbienne du sol (bactéries et champignons mycorhiziens) et les nématodes bactérivores: Effets sur la nutrition minérale et la croissance de différentes plantes. Dakar (SEN); Dakar: UCAD; IRD, 143 p.

22. Dodd JC (2000). The role of arbuscular mycorrhizal fungi in agro- and natural ecosystems. Outlook on Agriculture, 29(1), 55-62.

23. Egesi CN, Asiedu R, Ude G, Ogunyemi S, \& Egunjobi JK (2006). AFLP marker diversity in water yam (Dioscorea alata L.). Plant Genetic Resources, 4(3), 181-187. https://doi.org/10.1079/PGR2006121

24. El-Nagdi W, \& Youssef M (2004). Soaking faba bean seed in some bio-agents as prophylactic treatment for controlling Meloidogyne incognita root-knot nematode infection. Journal of Pest Science, 77, 75-78. https://doi.org/10.1007/s10340-003-0029-y

25. Ettien JB, Tschannen AB, Nindjin C, Asiedu R, Assa A and Girardin O (2003). Evaluation de nouvelles variétés de d'igname en Côte d'Ivoire : Bilan de trois années d'expérience avec des génotypes améliorés par l'IITA. Agronomie Africaine Numéro spécial (4): 109116.

26. Ezeh N (1998). Economics of Yam production and Postharvest Technology. In: Orkwor, G.C, R Asiedu and I.J Ekanayake eds. Food Yams: Advances in Research, IITA, Ibadan and NRCRI, Umudike, Nigeria. pp187-214.

27. FAO (1999). Culture et utilisation de l'igname pour assurer pour assurer la sécurité alimentaire, texte de référence, 1999, « http // www.fao.org » consulté le 10 juin 2015.

28. Fortuner R, \& Merny G (1973). Les nématodes parasites des racines associés Au riz en Basse-Casamance et en Gambie. Cahier ORSTOM, Serie Biologie, 21, 3-20.

29. Francl, L. (1993). Interaction of nematodes with mycorrhizae and mycorrhizal fungi in nematode interactions (ed M. W. Khan), chapman and Hall, New York, pp. 203-216. 
30. Hamel C. (1996). Prospects and problems pertaining to the management of arbuscular mycorrhizae in agriculture. Agriculture, Ecosystems and Environmen, 60, 197-210.

31. Hol WHG, \& Cook R (2005). An overview of arbuscular mycorrhizal fungi-nematode interactions. Basic and Applied Ecology, 6(6), 489503. https://doi.org/10.1016/j.baae.2005.04.001

32. Houedjissin RC, \& Koudande DO (2010). Etat des lieux de la recherche sur l'igname au Bénin. IITA, Rapport final.

33. Hussey R, \& Roncadori R (1982). Vesicular-Arbuscular mycorrhizae may limit nematode activity and improve plant growth. Plant Disease, 66, 9-14.

34. IIITA (International Institute of Tropical Agriculture). (2006). Plant Health Management Annual Report. Ibadan, Nigeria. Balaban Publishers.

35. ii. Ijoyah MO (2011). Yield effects of intercropping white guinea yam (Dioscoreae rotundata P.) minisetts and maize (Zea mays L.) in the southern Guinea savanna of Nigeria. African Journal of Agricultural Research, 6(23), 5227-5231. https://doi.org/10.5897/AJAR10.1135

36. Ile EI, Craufurd PQ, Asiedu R, \& Battey NH (2007). Duration from vine emergence to flowering suggests a long-day or rate of change of photoperiod response in white yam (Dioscorea rotundata P.). Environmental and Experimental Botany, 60, 86-94. https://doi.org/10.1016/j.envexpbot.2006.06.009

37. Jamani Y, Nyaku ST, Lutuf H, \& Cornelius E (2016). Plant-Parasitic Nematodes Associated with Yam in the Two Agro-Ecological Zones of Ghana. Nigerian Journal of Nematology, 3, 1-12.

38. Luc M, \& Coomans A (1992). Les nematodes phytoparasites du genre Xiphinema (Longidoridae) en Guyane et en Martinique. Belgique Journal of Zoology, 122(2), 147-183.

39. Morais CADM, Muniz FMDS, Lima( RS., Filho GM, \& Castro JMC (2016). Organic-matter effects on populations of dry rot of yam nematodes. African Journal of Agricultural Research, 11(17), 14941498. https://doi.org/10.5897/AJAR2016.10878

40. N'goran KE, Zohouri PG, Yoro RG, Kouakou MA, Assa A and Asiedu R (2007). Revue bibliographique sur la gestion de la fertilité des sols cultives en igname en Côte d'Ivoire, Agronomie Africaine 19 (3) : 281 - 288 (2007)

41. Nindjin C, Agbo N, Otokore D, Bricas N, Farah Z, Girardin O, \& Interjurassienne FR (2007). Les variétés d'igname (Dioscorea Spp.) rencontrées sur les marchés en Cote d'Ivore et leur préférence 
culinaire. Annales Des Sciences Agronomiques Du Bénin, 9(2), 1-12. https://doi.org/10.4314/asab.v9i2.43231

42. Nweke E, Okorjl C, Njoku E, \& King D. (1992). Elasticities of demand for major food items in a root and tuber crop-based food system. Emphasis on yam and cassava in southeast Nigeria. Resource and Crop Management Program. Res. Monogr. No.2, International Institute of Tropical Agriculture, Ibadan, Nig.

43. Nweke FI (2015) Yam in West Africa: Food, money, and more. Michigan State University Press. 167p.

44. Ojeniyi S (1990). Effect of Bush Clearing and Tillage Methods on Soil Physical and Chemical Properties of Humid Tropical Alfisols. Soil and Tillage Research, 15, 269-277.

45. Phillips J, \& Hayman D (1970). Improved procedures for clearing roots and staining parasitic and vesicular-arbuscular mycorrhizal fungi for rapid assessment of infection. Transactions of the British Mycological Society, 55(1), 158-161. https://doi.org/10.1016/S00071536(70)80110-3

46. Quénéhervé P (1998). Les Nematodes de l'igname, Cigname: Plante séculaire et culture d'avenir Berthaud, N. Bricas et 1.-L. Marchand éd.), Actes du séminaire international CIRAD-INRA-ORSTOMCORAF (3-6 Juin 1997, Montpellier).

47. Ravi V, Aked J, \& Balagopalan C (1996). Review on Tropical Root and Tuber Crops I . Storage Methods and Quality Changes. Critical Reviews in Food Science and Nutrition, 36(7), 661-709. https://doi.org/10.1080/10408399609527744

48. Ryan MH, \& Angus JF (2003). Arbuscular mycorrhizae in wheat and field pea crops on a low P soil : increased Zn-uptake but no increase in P-uptake or yield. Plant and Soil, 250, 225-239.

49. Senghor K. (1998). Etude de I'încidence du nématode phytoparasite Meloidome iavanica sur la croissance et ia symbiose fixatrice d'azote de douze espèces d'Acacia (Africains et Australiens) et mise en évidence du rôle des symbiotes endo et ectomycorhiziens contre ce nématode.

50. Siddiqi M. (2000). Tylenchida Parasites of Plants and Insects, 2nd Edition. CABI Publishing: Wallingford, UK.

51. Smith SE \& Read DJ (2008). Mycorrhizal symbiosis. 3rd Ed. Academic press. Academic Press.

52. Tchabi A, Burger S, Coyne D, Hountondji F, Lawouin L, Wiemken A, \& Oehl F (2009). Promiscuous arbuscular mycorrhizal symbiosis of yam ( Dioscorea spp .), a key staple crop in West Africa. Mycorrhiza, 19, 375-392. https://doi.org/10.1007/s00572-009-0241-6 
53. Tchabi A, Coyne D, Hountondji F, Lawouin L, Wiemken A, \& Oehl F (2008). Arbuscular mycorrhizal fungal communities in sub-Saharan Savannas of Benin, West Africa, as affected by agricultural land use intensity and ecological zone. Mycorrhiza, 18, 181-195. https://doi.org/10.1007/s00572-008-0171-8

54. Tchabi A, Coyne D, Hountondji F, Lawouin L, Wiemken A, \& Oehl F (2010). Efficacy of indigenous arbuscular mycorrhizal fungi for promoting white yam (Dioscorea rotundata) growth in West Africa. Applied Soil Ecology, 45(2), 92-100. https://doi.org/10.1016/j.apsoil.2010.03.001

55. Vestberg M, \& Kukkonen S (2004). Microbial inoculation for improving the growth and health of micropropagated strawberry. Applied Soil Ecology, 27, 243-258. https://doi.org/10.1016/j.apsoil.2004.05.006 